\title{
Application of the Model Predictive Control with Constraint Tightening for ATO System
}

\author{
Longsheng Wang ${ }^{1,2^{*}}$, Hongze $\mathrm{Xu}^{1}$, Changfu $\mathrm{Zou}^{3}$ and Guang Yang $^{1}$ \\ 1. School of Electronic and Information Engineering, Beijing Jiaotong University, \\ Beijing 100044, China \\ 2. Architecture and Traffic Engineering College, Guilin University of Electronic \\ Technology, Guilin 541004, China \\ 3. Department of Mechanical Engineering, University of Melbourne, Victoria \\ 3010, Australia \\ *lshwang@bjtu.edu.cn
}

\begin{abstract}
This paper addresses an optimal train trajectory planning and tracking problem for automatic train operation (ATO) with consideration of the train model uncertainty and constraints. Based on the discrete linear multi-points train model, an ATO control algorithm is presented to track piecewise reference by using model predictive control with constraints tightening such that the feasibility and robust convergence of this algorithm are guaranteed under the varying running resistance, automatic train protection (ATP) constraint, and train motor physical limits. Specifically, the features of the algorithm are: (i) taking traction and braking force of locomotives and braking force of carriges into account explicitly; (ii) integrating constraints tightening approach into piecewise reference tracking problem to ensure robustness; (iii) combining the optimal planning level and tracking control level together. Finally two case studies are conducted to verify the effectiveness of the algorithm.
\end{abstract}

Keywords: Train control system, automatic train operation (ATO), model predictive control (MPC), robustness, piecewise reference tracking

\section{Introduction}

Due to the advantages of high speed and bulk volume transportation, safe and comfortable riding, the rapid development of High-speed railway industry is witnessed worldwide over the past decades, which, however, results in the increasing demands in energy saving, safety and operational requirements. Hence it is necessary to develop an advanced, reliable and effective train control system (TCS) in terms of both meeting these requires above and dealing with nonlinear property of the train model. Automatic train operation (ATO), as an important subsystem of TCS, plays an important role in achieving optimal speed trajectory tracking with considering the safety, comfort and efficient objectives in the presence of the model uncertainty and external bounded disturbance.

Actually, dozens of optimal methods and advanced control algorithms had been used for the train operation problem over the recent decades [1-9]. First some energy-efficient operation strategies, which choose the proper switch points among the four driving phase (acceleration, cruising, coasting and braking) in order to minimize the energy cost for a single train, had been described [1,2]. On this basis some extended work have been presented, like the local to global energy-efficient control strategy with the local steep rail track in [3] and the multi-stage energy-efficient subway control approach for multi-trains [4]. Moreover, a number of advanced control methods had been used for train tacking problem, which can make sure not only the punctuality but also precise train stopping 
under the train overspeed protection, Linear Quadratic Regulation (LQR) [5], H2/Hळ control [6], robust adaptive control [7], iterative learning control [8]. Besides, in recent five years more and more work $[7,9]$ had been paid attention to safe constraints, which may bring the disasters if ignore them. The safe constraints contain the actuator saturation, speed limits, in-train forces, vertical and horizontal vibration As a result, it is very necessary that integrating the safe constraints into the process of problem formulation.

All of the methods above, however, are either only considering the optimal speed trajectory planning [1-3] or closed-loop tracking problem [5-8]. Compared with the two-level approach above, an approach combining the calculation of optimization with implementation of tracking control together is more cost effective. Furthermore, since the single-point train model $[1-2,4,8]$ could not implement traction and/or braking force of each car explicitly, using multi-points model is useful when the research focuses on the relative forces between the adjacent cars, like fault-tolerant control [7], optimal train operation [5].

The mentioned issues above can all be explicitly considered by model predictive control (MPC). MPC is a feedback control scheme in which an optimal control problem will be solved in each predictive horizon and only the first solution of the control sequence is implemented, then repeats this process again in the next predictive horizon [10]. More importantly, the feature of MPC, which is dealing with constraints control problem naturally and allowing the operation closer to constraints, is effective for the train motor physical constraints. Moreover, compared with other robust approaches ( LMIs and min-max robust MPC), constraint tightening [11] is an efficient robust method which avoids computation complexity by using only a nominal prediction model and constraints recursion algorithm to achieve robustness.

In this paper, a discrete linear multi-points train model is used for taking the distributed traction and/or braking force of each car into account, and an ATO algorithm based on model predictive control with constraint tightening (CTMPC) is proposed for tracking piecewise train speed profile under the ATP curve in the presence of bounded disturbances, constraints and model mismatch.

The reminder of this paper is organized as follows. Section 2 introduces the multi-points train model based on the discrete linear state-space equation. Then the optimal trajectory planning and control problem under the consideration of constraints is formulated in section 3. In section 4 an algorithm using constraints tightening MPC is proposed for optimal train operation. Section 5 illustrates the simulation results of two cases. Concluding remarks are given in section 6 .

\section{Train Multi-Points Model}

In this section, for the sake of simplicity some notations and symbols are presented first. The multi-points train model, which consists of cascade locomotives and carriages by the spring coupling, is reviewed. Then the discrete linear state-space model of train in the presence of the unknown and bounded disturbances is proposed for the MPC design.

\subsection{Notations and Symbols}

For a better understanding, the notations and symbols used in the paper are introduced as follows:

- The set operator “ " denotes the Pontryagin difference [12]

$$
\mathcal{A} \sim \mathcal{B}=\{a \mid a+b \in \mathcal{A}, \forall b \in \mathcal{B}\},
$$

which deduces an important property will be used in the proof of this paper.

$$
a \in(\mathcal{A} \sim \mathcal{B}), b \in \mathcal{B} \Rightarrow(a+b) \in \mathcal{A} .
$$


- The multiplication of a matric $A$ and a set $\mathcal{B}$ is defined as:

$$
A \mathcal{B}=\{c \mid \exists b \in \mathcal{B}, c=A b\} .
$$

- The notation $(j ; k)$ denotes the prediction state or input at the $j$ steps based on the state at time step $k$; similarly $(j ; k+1)$ is a the notation based on time step $k+1$.

- The symbols $\mathbf{I}_{n \times m}$ and $0_{n \times m}$ are the $n \times m$ identity matrix and zero matrix respectively.

- The superscript "0" denotes the optimal solution to the optimization problem in the following.

- The symbol $\|$.$\| is defined as the two-norm, hence \|x\|_{Q}:=x^{T} Q x$.

-The notation $(\mathbf{a}, \mathbf{b}):=\left[\mathbf{a}^{T}, \mathbf{b}^{T}\right]^{T}$, in which $\mathbf{a}, \mathbf{b}$ are both vectors.

\subsection{Train Dynamics}

The discrete linear time-variant high-speed train model that will be linearized at each sampling time is described for model predictive controller design, which is based on a similar multi-points train model first proposed by Xia in [13] for heave-haul trains.

Without loss of generally, due to the Newton's law the dynamics of the train, which is connected by locomotives and wagons through the springs, can be described as:

$$
\left\{\begin{array}{l}
m_{i} \dot{v}_{i}=u_{i}+f_{i n_{(i-1) i}}-f_{i n_{i(i+1)}}-F_{r_{i}}, \quad(i=1,2, \ldots, n), \\
\dot{x}_{i}=v_{i},
\end{array}\right.
$$

where $x_{i}, v_{i}$ and $m_{i}$ are the displace, speed and mass of the $i_{t h}$ car respectively, hence $\dot{v}_{i}$ is the acceleration or deceleration of the $i_{t h}$ car; and $u_{i}$ is the train traction or braking force of each car, $f_{i_{(i-1) i}}$ denotes the in-train coupling force between two adjacent $(i-1)_{t h}$ and $i_{t h}$ cars, $F_{r_{i}}$ is the resistance force of each car. Notes that for the first and last car, there is only one coupling force, hence $f_{i n_{01}}=0$ and $f_{i n_{n(n+1)}}=0$. The variable $n$ is the total number of the cars, including locomotives and wagons.

Some details about the in-train coupling force and resistance force are reviewed here. For one thing, the in-train force is mainly divided into two parts: spring part and damping part, expressed as:

$$
f_{i n_{(i-1) i}}=k\left(x_{i-1}-x_{i}\right)+d\left(\dot{x}_{i-1}-\dot{x}_{i}\right),
$$

where $k$ and $d$ denote the spring and damping coefficients respectively. Furthermore, it is almost impossible to obtain a precise equation to describe the resistance force, so an empirical equation is used:

$$
F_{r_{i}}=m_{i}\left(c_{0}+c_{v} v_{i}+c_{a} v_{i}^{2}\right)+g\left(x_{i}\right),
$$

where the resistance force of each car consists of the additional resistance force $g\left(x_{i}\right)$ and the running resistance force $m_{i}\left(c_{0}+c_{v} v_{i}+c_{a} v_{i}{ }^{2}\right)$, and $c_{0}, c_{v}, c_{a}$ denote the resistance coefficients. It is apparent that the nonlinear property of the resistance force will account for the important part with the increasing of the train speed. As well in practice the resistance coefficients are not constants all the time, they may even be some time-varying parameters. Besides, the additional resistance force (including gradient, tunnel and curve resistance force) is also not easy to be described in a mathematical form such that it will be regarded as an unknown and bounded disturbance. Therefore the equation (4) is rewritten as: 


$$
\begin{array}{r}
m_{i} \dot{v}_{i}=u_{i}+k\left(x_{i-1}-2 x_{i}+x_{i+1}\right)+d\left(v_{i-1}-2 v_{i}+v_{i+1}\right) \\
-\left(m_{i}\left(c_{0}+c_{v} v_{i}+c_{a} v_{i}{ }^{2}\right)+g\left(x_{i}\right)\right),
\end{array}
$$

Sequentially, a new state $\mathbf{x}:=\left[v_{1}, v_{2}, \cdots, v_{n}, x_{1}, x_{2}, \cdots, x_{n}\right]^{T}$ and the new input $\mathbf{u}:=\left[u_{1}^{\prime}, u_{2}^{\prime}, \cdots, u_{n}^{\prime}\right]^{T}$ is defined for transforming the equation (7) to a standard state-space form, where the new input $u^{\prime}$ is the deviation of the real input and steady-state input: $u^{\prime}:=u_{i}-u_{s}$. The system will be linearized at each operating point $\theta:=\left(\mathbf{u}_{s}, \mathbf{x}_{s}\right)$, where $\mathbf{u}_{s}$ and $\mathbf{x}_{s}$ denote the steady states and inputs of the system, and satisfy the equation (8):

$$
\left(A-\mathbf{I}_{n}\right) \mathbf{x}_{s}+B \mathbf{u}_{s}=\mathbf{0}_{n}
$$

Besides, $\mathbf{w} \in \mathcal{W}$ denotes an unknown and bounded external disturbance that come from the additional resistance forces from equation (6). Therefore the result of linearizing equation (7) using Taylor series at operation point is:

$$
\begin{array}{r}
m_{i} \dot{v}_{i}=\left(u_{i}-u_{s}\right)+k\left(x_{i-1}-2 x_{i}+x_{i+1}\right)+d\left(v_{i-1}-2 v_{i}+v_{i+1}\right) \\
\left.-\left(m_{i}\left(c_{v}+2 c_{a} v_{s}\right) v_{i}\right)+g\left(x_{i}\right)\right),
\end{array}
$$

which is rewritten as the metrics form

$$
\dot{\mathbf{x}}=A_{p} \mathbf{x}+B_{p} \mathbf{u}+\mathbf{w},
$$

where

$$
\begin{aligned}
& \mathbf{w}=-\left[\frac{g\left(x_{1}\right)}{m_{1}}, \frac{g\left(x_{2}\right)}{m_{2}}, \cdots, \frac{g\left(x_{n}\right)}{m_{n}}\right]^{T}, \\
& A_{p}=\left[\begin{array}{cc}
A_{11} & A_{12} \\
\mathbf{I}_{n \times n} & \mathbf{0}_{n \times n}
\end{array}\right] \text {, } \\
& B_{p}=\left[B_{1}, \mathbf{0}_{n \times n}\right]^{T} \text {, } \\
& B_{1}=\operatorname{diag}\left(1 / m_{1}, \ldots, 1 / m_{n}\right)_{n \times n}, \\
& A_{111}=\left[\begin{array}{cccccc}
\frac{d}{m_{1}} & -\frac{d}{m_{1}} & 0 & \cdots & 0 & 0 \\
\frac{d}{m_{2}} & -2 \frac{d}{m_{2}} & \frac{d}{m_{2}} & \cdots & 0 & 0 \\
\vdots & \vdots & \vdots & \ddots & \vdots & \vdots \\
0 & 0 & \cdots & \frac{d}{m_{n-1}} & -2 \frac{d}{m_{n-1}} & \frac{d}{m_{n-1}} \\
0 & 0 & \cdots & 0 & \frac{d}{m_{n}} & -\frac{d}{m_{n}}
\end{array}\right]_{n \times n} \\
& A_{112}=-\operatorname{diag}\left(c_{v}+2 c_{a} v_{0}, c_{v}+2 c_{a} v_{0}, \cdots, c_{v}+2 c_{a} v_{0}\right) \text {, } \\
& A_{11}=A_{111}+A_{112} \text {, } \\
& A_{12}=\left[\begin{array}{cccccc}
\frac{k}{m_{1}} & -\frac{k}{m_{1}} & 0 & \cdots & 0 & 0 \\
\frac{k}{m_{2}} & -2 \frac{k}{m_{2}} & \frac{k}{m_{2}} & \cdots & 0 & 0 \\
\vdots & \vdots & \vdots & \ddots & \vdots & \vdots \\
0 & 0 & \cdots & \frac{k}{m_{n-1}} & -2 \frac{k}{m_{n-1}} & \frac{k}{m_{n-1}} \\
0 & 0 & \cdots & 0 & \frac{k}{m_{n}} & -\frac{k}{m_{n}}
\end{array}\right]_{n \times n},
\end{aligned}
$$

Finally, the continuous state-space equation (10) is discretized with sampling time $T_{s}$ to the form bellow:

$$
\mathbf{x}(k+1)=A(k) \mathbf{x}(k)+B \mathbf{u}(k)+\mathbf{w}(k),
$$




\section{Problem Formulation}

In this section the optimal objectives of minimizing the speed-displace tracing error, energy consumption, in-train force as well as confirming a comfortable journey is presented, which are integrated into a cost function with appropriate penalty weightings. Subsequently the constraints including the physical limits and ATP speed limits and definition of admissible maximal invariant set are introduced. Hence the train operation optimal problem, which not only meets the optimal objectives above under consideration of the protection of ATP curve but also deal with the robustness despite of the bounded disturbance, is formulated.

\subsection{Stage Cost Function}

The objective of the paper is designing an automatic train operation strategy which renders the speed and position of the train to track a desired speed-position profile which is obtained a priori by the operation schedule system in order to meet the needs of punctuality, as well as reduce the change rate of tracking or braking force $\Delta \mathbf{u}^{T} R^{\prime} \Delta \mathbf{u}$ for a comfortable travel, sum of tracking and braking force $\mathbf{u}^{T} R \mathbf{u}$ for energy saving and in-train force $\mathbf{x}_{i n}^{T} Q_{i n} \mathbf{x}_{i n}$ for safe. So the stage cost function will be formulated as:

$$
\ell=Q_{x}\left(x_{1}-x_{d}\right)^{2}+Q_{v}\left(v_{1}-v_{d}\right)^{2}+\Delta \mathbf{u}^{T} R^{\prime} \Delta \mathbf{u}+\mathbf{u}^{T} R \mathbf{u}+\mathbf{x}_{i n}^{T} Q_{i n} \mathbf{x}_{i n},
$$

where $Q_{x}, Q_{v}, Q_{i n}, R, R^{\prime}$ are all positive definite matrices denote the weighting penalty for displace, speed, in-train force, energy and trip comfort respectively, and $\mathbf{x}_{\text {in }}:=\left\{x_{1}-x_{2}, x_{2}-x_{3}, \cdots, x_{n-1}-x_{n}\right\}^{T}$, hence $\mathbf{x}_{\text {in }}=Z \mathbf{x}$.

In order to transform the stage cost function (12) to a standard cost form, the artificial state of the train $\mathbf{x}_{s}(k):=\left\{v_{d}(k), x_{d}(k), 0, \cdots, 0\right\}^{T}$, which consists of desired speed $v_{d}(k)$ and displace $x_{d}(k)$ profile, and state weights $Q:=\operatorname{diag}\left\{Q_{v}, Q_{x}, Z^{T} Q_{i n} Z\right\}$ are defined. Hence (12) is rewritten as:

$$
\begin{aligned}
\ell(\mathbf{x}(k), \mathbf{u}(k))= & \left\{\left(\mathbf{x}(k)-\mathbf{x}_{s}(k)\right)^{T} Q\left(\mathbf{x}(k)-\mathbf{x}_{s}(k)\right)\right. \\
& \left.+\Delta \mathbf{u}(k)^{T} R^{\prime} \Delta \mathbf{u}(k)+\mathbf{u}(k)^{T} R \mathbf{u}(k)\right\},
\end{aligned}
$$

Remark 1 (Physical Meaning). The target state $\hat{\mathbf{x}}_{s}$ in the train operation is just the ATP curve, which means the real state $\mathbf{x}(k)$ will be as close as possible to the target $\hat{\mathbf{x}}_{s}$ for an optimal operation but could not touch it because the train will be forced to stop if the real speed of the train excesses the ATP protection speed. Additionally, the artificial steady-state $\left(\mathbf{x}_{s}, \mathbf{u}_{s}\right)$ form equation (8) denotes an ideal speed-displace profile which satisfies the optimal objectives under the protection of ATP. Therefore, the optimal train operation is tracking the artificial steady-state $\left(\mathbf{x}_{s}, \mathbf{u}_{s}\right)$ in presence of the target $\hat{\mathbf{x}}_{s}$ constraint and bounded disturbance $\mathbf{w} \in \mathcal{W}$.

\subsection{Constraints}

As mentioned in introduction, we could not ignore the constraints which may cause a vital accident. The constraints mainly consist of three parts: the first one is the actuator saturation of the train motor or braking system and changes of them which come from the mechanical characteristic; the second one is ATP speed limits due to the safe protection or emergency requires for running schedule; the last one is the tolerance bound of the in-train forces. That is, the input and state must satisfy the following constraints equations: 


$$
\left\{\begin{array}{l}
u^{\min } \leq u_{i} \leq u^{\max }, \\
\Delta u^{\min } \leq \Delta u_{i} \leq \Delta u^{\text {max }} \\
0 \leq v_{i} \leq v^{\text {max }} \\
f_{\text {in }}^{\text {min }} \leq f_{\text {in }_{(i-1) i}} \leq f_{i n}^{\max }
\end{array}\right.
$$

where the superscript max, min denote the upper and lower bound of the value respectively, and the operator $\Delta$ is defined as $\Delta(\cdot)=(\cdot)(k)-(\cdot)(k-1)$. Specifically, when $u>0$, $\mathrm{u}$ comes from the tracking force, so $u^{\max }$ and $\Delta u^{\max }$ depend on the mechanical characteristics of the motor. Similarly, the braking systems determine the value of $u^{\text {min }}$ and $\Delta u^{m i n}$. And it must transfer to the form of $u^{\prime}$. As well due to (), the constraints of $f_{i n_{i}}$ can be transformed to the constraints of system state $x_{i n_{i}}$. Additionally, as $\Delta u_{s}=c_{a}\left(v_{k}^{2}-v_{k-1}^{2}\right)$ and the rate of train speed is small, $\Delta u^{\prime}=\Delta u-\Delta u_{s} \approx \Delta u$. Hence the equation (10) is expressed as:

$$
\left\{\begin{array}{l}
\left(u^{\text {min }}-u_{s}\right) I_{n \times 1} \leq \mathbf{u} \leq\left(u^{\max }-u_{s}\right) I_{n \times 1}, \\
\Delta u^{\text {min }} I_{n \times 1} \leq \Delta \mathbf{u} \leq \Delta u^{\max } I_{n \times 1}, \\
E^{\text {min }} \leq \mathbf{x} \leq E^{\text {max }},
\end{array}\right.
$$

where $E^{\text {min }}=\left[0, \cdots, 0, \frac{f_{i n}^{\min }}{k}, \cdots, \frac{f_{i n}^{\min }}{k}\right]$ and $E^{\max }=\left[v^{\max }, \cdots, 0, \frac{f_{i n}^{\max }}{k}, \cdots, \frac{f_{i n}^{\max }}{k}\right]$.

Finally, a constraint output $\mathbf{y}$ is defined for integrating all the state and input constraints together into the output constraint $\mathcal{Y}$ for the sake of progress of constraints tightening.

$$
\mathbf{y}(k):=C \mathbf{x}(k)+D \mathbf{u}(k) \in \mathcal{Y}
$$

where $C$ and $D$ are chosen by the designer arbitrarily. Without loss of generality, the most common method is choosing proper $C$ and $D$ to render output $\mathbf{y}$ to identity.

\subsection{The Maximal Admissible Invariant Set}

The maximal admissible invariant set [14] for robust tracking problem is reviewed here, which will be used as the state terminal constraint of CTMPC problem. Consider the particular controller for system (11),

$$
\kappa(x, \theta)=K x+L \theta,
$$

and the augmented state vector $\mathbf{z}:=(\mathbf{x}, \theta)$ for the augmented system $\mathbf{z}^{+}=A_{z} \mathbf{z}$ where

$$
A_{z}=\left[\begin{array}{cc}
A+B K & B L \\
0 & I_{n}
\end{array}\right]
$$

So the maximal admissible invariant set is expressed as:

$$
\mathcal{O}_{\infty, \lambda}^{z}=\left\{z: A_{z}^{i} z \in \mathcal{Z}_{\lambda}, \forall i \geq 0, \lambda \in(0,1)\right\}
$$

where $\mathcal{Z}_{\lambda}=\{z:(x, K x+L \theta) \in \Omega, \theta \in \lambda \Omega\}$

\subsection{MPC Optimization Problem Formulation}

In summary the MPC optimization problem $P_{N}\left(x_{k}, \hat{\mathbf{x}}_{s}, \mathcal{Y}, \mathcal{W}\right)$, which penalizes the deviation from the ideal train reference state and input $\left(\mathbf{x}_{s}, \mathbf{u}_{s}\right)$, and the deviation between the ideal train reference state and ATP constraints $\hat{\mathbf{x}}_{s}$, as well as 
addresses the robustness using the constraint tightening, is formulated subject to the system dynamic (11), output constraints (16) and terminal set (19)

The cost function for problem $P_{N}\left(x_{k}, \hat{\mathbf{x}}_{s}, \mathcal{Y}, \mathcal{W}\right)$ based on [15] is defined as:

$$
V_{N}\left(x_{k}, \hat{\mathbf{x}}_{s}, \mathbf{u}, \theta\right)=\sum_{i=0}^{N-1} \ell(\mathbf{x}, \mathbf{u})+\left\|\mathbf{x}(N ; k)-\mathbf{x}_{s}\right\|_{P}^{2}+\left\|\mathbf{x}_{s}-\hat{\mathbf{x}}_{s}\right\|_{T}^{2}
$$

where $P$ and $T$ are positive definite matrices, and $P$ satisfy the equation below

$$
(A+B K)^{T} P(A+B K)-P=-\left(Q+K^{T} R K\right)
$$

Note that the terminal cost term here is much different from the common terminal term, as sometimes the target steady-state is not accessible but artificial steady-state is always accessible, the alternate solution is using the sum of deviation between prediction terminal state and artificial state and deviation between artificial state and target state.

As a result, the optimal problem $P_{N}\left(x_{k}, \hat{\mathbf{x}}_{s}, \mathcal{Y}, \mathcal{W}\right)$ with consideration of the tightening set $\mathcal{Y}$ and the bounded disturbance $\mathrm{w} \in \mathcal{W}$ is:

$$
\begin{array}{r}
V_{N}^{0}\left(x_{k}, \hat{\mathbf{x}}_{s}\right)=\min _{\mathbf{u}, \theta} V_{N}\left(x_{k}, \hat{\mathbf{x}}_{s}, \mathbf{u}, \theta\right) \\
\text { s.t. }(j=0,1, \cdots, N-1) \\
\mathbf{x}(j+1 ; k)=A \mathbf{x}(j ; k)+B \mathbf{u}(j ; k) \\
\mathbf{y}(j ; k)=C \mathbf{x}(j ; k)+D \mathbf{u}(j ; k) \in \mathcal{Y}(j) \\
\mathbf{x}(0 ; k)=x(k) \\
(\mathbf{x}(N), \theta) \in \mathcal{X}_{F}^{z}
\end{array}
$$

\section{ATO Algorithm Based on CTMPC}

This section proposed an ATO algorithm which is based on CTMPC for piecewise constant reference. In the meantime the CTMPC algorithm, proposed by Gossner et al. [16] and extended by Richards et al. [11], simplifies the complexity of the computation by using only a nominal model prediction model and changing the constraints to achieve robustness. Intuitively, the key idea is to retain a "margin" of the constraints for the future feedback. Hence the most important feature is combining the mature nominal MPC theory with a recursion tightening constraints technique.

The constraint tightening will be taking the influences of the disturbances into recursion of the model constraints set $\mathcal{Y}$, which guarantees the stability and robustness through the recursion constraints set $\mathcal{Y}(j)$. The recursion algorithm [11] is described as:

$$
\begin{aligned}
\mathcal{Y}(0) & =\mathcal{Y}, \\
\mathcal{Y}(j+1) & =\mathcal{Y}(j) \sim(C+D K(j)) L(j) \mathcal{W}, \quad(j=0,1, \cdots, N-1)
\end{aligned}
$$

where $K(j)$ and $L(j)$ are candidate control policy and state transition matrices respectively. As well the recursion algorithm of $L(j)$ is:

$$
\begin{aligned}
L(0) & =I, \\
L(j+1) & =(A+B K(j)) L(j) . \quad(j=0,1, \cdots, N-1)
\end{aligned}
$$

There are a number of possible methods for choosing a candidate control policy $K(j)$, such as polo placement candidate, nilpotent candidate, However the nilpotent 
candidate control policy which renders the state to origin is much benefit for simplicity the computation of the recursion. We assume that the state of the nominal system is driven to original at time step $M$, hence $L(j)=0, \quad(\forall j=M, M+1, \cdots, N)$, which makes the constraints set retain in a constant value after time step $M, \mathcal{Y}(j)=\mathcal{Y}(M), \quad(\forall j=M, M+1, \cdots, N)$. Therefore nilpotent policy is an effective and efficient candidate control policy due to the calculation of recursion is just applying in the first $M$ steps. The nilpotent algorithm is defined as:

$$
\begin{aligned}
& \text { Algorithm } 1 \text { (Nilpotent candidate policy) } \\
& \text { for } j=M-1:-1: 0 \text {, } \\
& P(M)=\infty \mathbf{I}, \\
& K(j)=-\left[R+B^{T} P(j+1) B\right]^{-1} B^{T} P(j+1) A, \\
& P(j)=Q+A^{T} P(j+1) A-A^{T} P(j+1) B\left[R+B^{T} P(j+1) B\right]^{-1} B^{T} P(j+1) A, \\
& \text { end } \\
& K(j)=0, \forall j>M .
\end{aligned}
$$

Another significant work for constraint tightening is choosing a terminal constraint set $\mathcal{X}_{F}$. To begin with, the admissible invariant set should satisfy the request below for tightening, that is,

$$
\mathcal{X}_{F}=\mathcal{R} \sim L(N) \mathcal{W},
$$

where $\mathcal{R}$ is a robust control invariant set, and a control law $\kappa(x, \theta)$ is selected in order to must meet the requires below,

$$
\begin{aligned}
& \forall x \in \mathcal{R}, \quad \forall w \in \mathcal{W} \\
& A x+B \kappa(x, \theta)+L(N) w \in \mathcal{R}, \\
& C x+D \kappa(x, \theta) \in \mathcal{Y}(N)
\end{aligned}
$$

Notes that if a nilpotent control candidate is chosen, because of the property of candidate control policy, $L(N)=0$, which can ignore the influence of $L(N)$ in the design of terminal set. It is another advantage for the nilpotent policy in terms of simplifying the computation.

Moreover, there are also numbers of methods which can make the set $\mathcal{R}$ satisfy (27), such as Maximal Robust Control Invariant set for the greatest feasible set, Maximal Robust Output Admissible set for robust tracking problem. The latter one is selected as the terminal constraints set in this paper. Use the invariant set in section 3, hence

$$
\mathcal{X}_{F}=\mathcal{O}_{\infty, \lambda}^{z}
$$

Finally the algorithm of robust model predictive control for tracking piecewise constant references using constraint tightening for the optimal problem (22) is described as: $k$

Algorithm 2 (CTMPC for tracking piecewise constant references):

step 1. Get the measurement (or estimation) value of the state $x(k)$ at current time

step 2. Formulate the MPC optimization problem $P_{N}\left(x_{k}, \hat{\mathbf{x}}_{s}, \mathcal{Y}, \mathcal{W}\right)$ subject to constraints in (22); 
step 3 . Compute the nilpotent candidate control policy $K(j)$ using algorithm (25) and state transition matrices $L(j)$ using (24);

step 4. After that the constraint tightening set $\mathcal{Y}$ for optimization problem $P_{N}\left(x_{k}, \hat{\mathbf{x}}_{s}, \mathcal{Y}, \mathcal{W}\right)$ is obtained by $(23)$;

step 5. Choose the suitable terminal set $\mathcal{X}_{F}$ based on (28);

step 6. Use nominal MPC method [10] to solve $P_{N}\left(x_{k}, \hat{\mathbf{x}}_{s}, \mathcal{Y}, \mathcal{W}\right)$, get the optimal sequence $\mathbf{u}^{0}:=\left\{u^{0}(0, x(k)), u^{0}(1, x(k)), \cdots, u^{0}(N-1, x(k))\right\}$, which the optimal sequence $\mathbf{u}^{0}=\arg _{\mathbf{u}} V_{N}^{0}\left(x_{k}, \hat{\mathbf{x}}_{s}\right)$;

step 7. Implement the controller just using the first term in the sequence $u(k):=u^{0}(0, \mathbf{x}(k))$;

step 8 . Repeat progress step. 1 to step.7 again at next sampling time $k+1$.

Theorem 1. Consider that the target steady state $\hat{\mathbf{x}}_{s}$ is admissible. Suppose that (28) hold for a given $\lambda \in(0,1)$ and stage cost function (13) hold. If the train (11) is controlled by the Algorithm 2 and subjected to constraints (22) and disturbance $\mathbf{w} \in \mathcal{W}$ under the automatic train protection and the optimal problem $P_{N}\left(\mathbf{x}_{0}, \hat{\mathbf{x}}_{s}, \mathcal{Y}, \mathcal{W}\right)$ is feasible at the initial state $\mathrm{x}_{0}=\mathbf{x}(k)$, then the following statements hold.

1) All subsequent optimal problem $P_{N}\left(\mathbf{x}_{k}, \hat{\mathbf{x}}_{s}, \mathcal{Y}, \mathcal{W}\right)$ are feasible;

2) The state of the train asymptotically converges to the following set

$$
\mathcal{X}_{C}=\left\{\mathrm{x} \in \mathcal{R}^{N} \mid\left\|\mathbf{x}-\hat{\mathbf{x}}_{s}\right\|_{T} \leq \alpha\right\}
$$

where $\alpha:=\max _{w \in \mathcal{W}} \sum_{i=0}^{N}\left(\|L(i) w\|_{Q}+\|K(j) L(j) w\|_{R}\right)$

Proof: See Appendix.

\section{Numerical Simulation}

In order to verify the performance of the proposed Algorithm 2 using CTMPC, two cases are presented in this section, of which the train for this simulation is CRH3.

Table 1. Parameters of CRH3

\begin{tabular}{c|c|c}
\hline \hline Property & Symbol & Values \\
\hline mass of locomotives $[\mathrm{kg}]$ & $m_{i}(i=1,3,6, \beta)$ & $4.5 \times 10^{1}$ \\
\hline mass of carriages $[\mathrm{kg}]$ & $m_{i}(i=2,4,5,7)$ & $5 \times 10^{1}$ \\
\hline coefficient of elasticity[N/m & $k$ & $2 \times 10^{7}$ \\
\hline $\begin{array}{c}\text { coefficient of damping[ } \\
N \times s / m]\end{array}$ & $d$ & $5 \times 10^{6}$ \\
\hline $\begin{array}{c}\text { coefficient of resistance[ } \\
N / \mathrm{kg}]\end{array}$ & $c_{0}$ & $5.2 \times 10^{-2}$ \\
\hline $\begin{array}{c}\text { coefficient of resistance[ } \\
(N \times s) /(m \times k g)]\end{array}$ & $c_{v}$ & $3.8 \times 10^{-3}$ \\
\hline $\begin{array}{c}\text { coefficient of resistance[ } \\
\left.\left(N \times s^{2}\right) /\left(m m^{2} \times \mathrm{kg}\right)\right]\end{array}$ & $c_{a}$ & $1.1 \times 10^{-4}$ \\
\hline \hline
\end{tabular}

There are eight cars (4 locomotives that have both traction and braking force and 4 carriages with only braking force) for CRH3 [17]. Here assume that the speed and the displace of each cars can be measured via sensors, equivalently full state vector 
of the system is available. However, the simulation will just use 3 cars model that includes 2 locomotives and one carriage, which can represent all the situations. As using 8 cars model not only increases the calculation but also is so difficult to distinguish in one figure. The simulation parameters of $\mathrm{CRH} 3$ for the simulation are shown in Table 1. Additionally, the constraints in section 3 are presented as follow. (i) Due to the physical limits of the traction unit, the maximum rated power $P_{\max }=550 \mathrm{kw}$, hence the $u_{\max }=P_{\max } / v(k)$. Similarly, the maximum rated power of braking unit is $u_{\min }$, hence the traction or braking force for the three cars model is limited by: $-200 k N \leq u_{1,3} \leq 200 k N$ and $-200 k N \leq u_{2} \leq 0 k N$; (ii) The maximal in-train force of the spring coupling for the sake of safety is $-1000 k N \leq \mathrm{f}_{i n} \leq 1000 k N$; (iii) The ATP profile is shown in Figure 1. (the red dashed line); (iv) For a comfort journey, the rate of traction and braking force is restricted in $\Delta u \leq 50 \mathrm{kN}$.

Besides, the parameters of CTMPC are given as follows. Sampling time $T_{s}=1 \mathrm{~s}$, prediction and control horizon are $N_{p}=10$ and $N_{u}=3$, weightings in states and inputs $\quad$ are $\quad Q=\operatorname{diag}\{1000,1000,1000,0,0\} \quad, \quad R=\operatorname{diag}\{0.1,0.1,0.1\} \quad$ and $R^{\prime}=\operatorname{diag}\{0.1,0.1,0.1\}$ respectively, $\lambda=0.99$ and $T=100 P$. The Matlab MPC toolbox, MPT3 [18] and invariant set toolbox [19] are using in the simulation. Two cases are considered in this paper:

\subsection{Case A}

In case A, the nominal train model is chosen as the simulation model, since we will consider the tracking performance for the piecewise reference under Algorithm 2 firstly. In this case study, the ATP profile is a piecewise reference (see Figure 1), which means there are different speed limits in different track section. Such as, a train should run in a low speed for the sake of safety when it is crossing a village or the urban area of a city. In Figure 1, the speed limits of ATP (red dashed line) are 20m/s ( $0-60 \mathrm{~s}), 50 \mathrm{~m} / \mathrm{s}(60 \mathrm{~s}-$ $240 \mathrm{~s})$ and $30 \mathrm{~m} / \mathrm{s}(240 \mathrm{~s}-300 \mathrm{~s})$ respectively. Note that in the time $210 \mathrm{~s}-240 \mathrm{~s}$, the ATP profile remains some margins for train braking in the braking phase.

Consequently, the simulation results under case A are showing in Figure 1, Figure 2 and Table 2. In this case study, the weightings in speed error is penalized more than weighting in energy saving for a schedule objective. The green dots line in Figure 1 represents the artificial steady states, equivalently a reference under the optimal objective, and the blue dash-dot line is the real states under the Algorithm 2. It is apparent that artificial curve satisfies the optimal objective under the ATP protection, and then the real state will track it as well almost without any error, which proves that automatic train operation under Algorithm 2 have a good tracking performance.

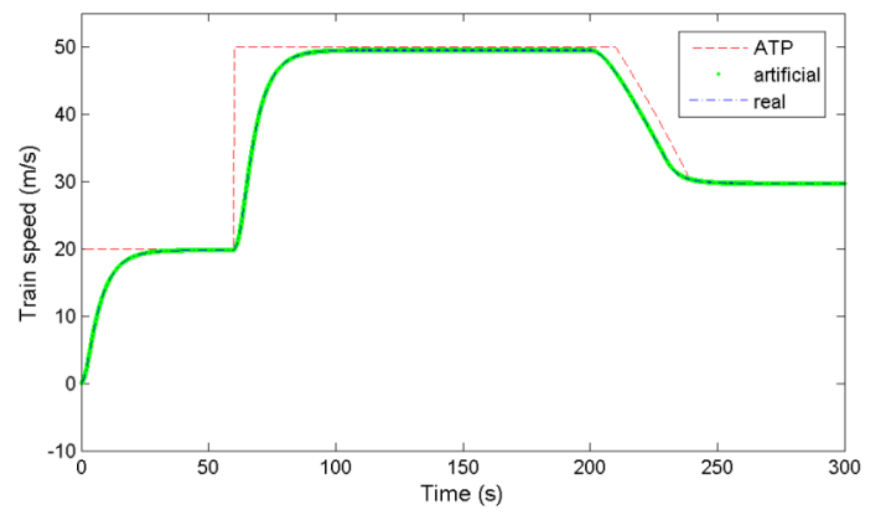

Figure 1. Train Speed Trajectory under Case a for Train Operation 
Furthermore, the Figure 2 displays the traction and braking force of the three cars ( $\mathrm{u} 1, \mathrm{u} 2, \mathrm{u} 3$, respectively). As the second car only equipped with a braking unit, the traction force is always zero and braking force just exists in braking phase (see 112 in Figure 2). In contrast, the first and third cars equipped with both traction and braking units are almost same from beginning to the end. Note that at time 70s, the traction force reaches the upper bound $200 \mathrm{kN}$ and retains in this bound for a while, which verifies the most important feature of MPC - performance for addressing constraints problem. Additionally, it is evident that the in-train force always remain in the same range from the Table 2 which shows the in-train force between the adjacent cars.
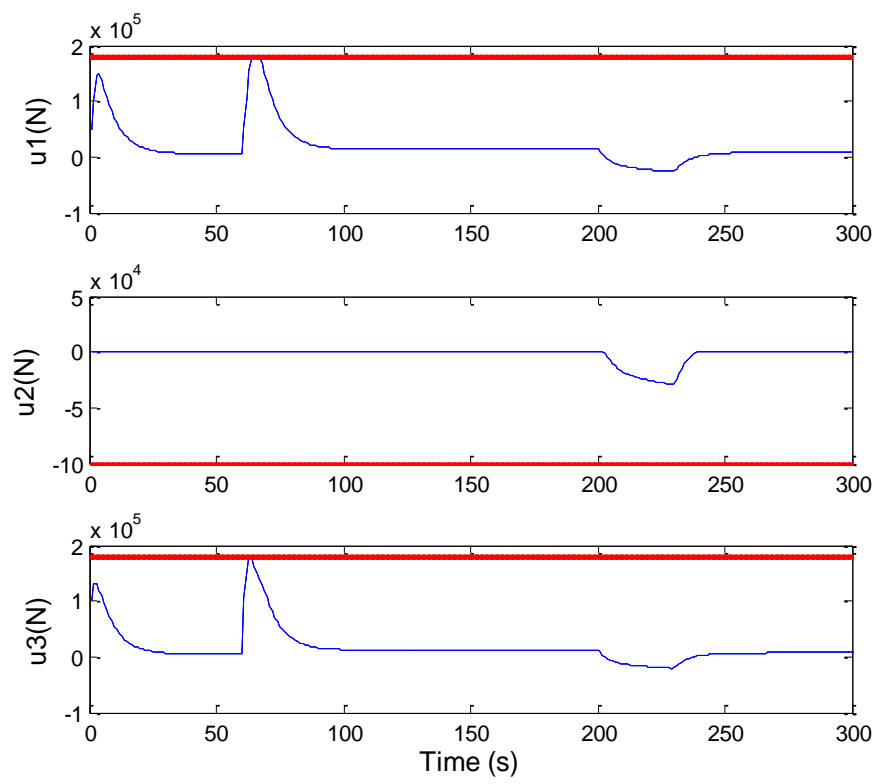

Figure 2. Traction/Braking Force under Case A

Table 2. In-Train Force under Case A

\begin{tabular}{c|c|c|c}
\hline \hline & Mean & Max & Min \\
\hline$f_{i n_{1}}[k N]$ & 6.23 & 70.7 & -24.9 \\
\hline$f_{\text {in. }_{2}}[k N]$ & -7.52 & 25.1 & -79.7 \\
\hline \hline
\end{tabular}

\subsection{Case B}

In case $\mathrm{B}$, the simulation model under the consideration of bounded unknown disturbance is used. We assume that the bound of additional force in (6) is $1000 \mathrm{~N}$, hence the bound of disturbance $\mathbf{w}$ in (11) is approximate 0.02 . Therefore $w_{1}, w_{2}, w_{3}$ are chosen as bounded white noise with different seeds in this simulation. And the parameter in nilpotent policy is $M=3$, other details are same as case A.

The result in Figure 3 is the train speed under case B without using constrains tightening. We find that the real states have exceeded the ATP curve in some time in the presence of the disturbance, which may lead to unnecessary braking or emergency stop under the protection of train operation system. There is no doubt that both braking and stop are not what we want as they not only waste energy but also influence the timetable.

In contrast, Figure 4 shows the train speed under case B when using constraints tightening, namely Algorithm 2. Evidently, while the real train speed is a little less than it 
in Figure 4, it is entirely under the ATP curve. Correspondingly, compared with Figure 3 the traction and braking

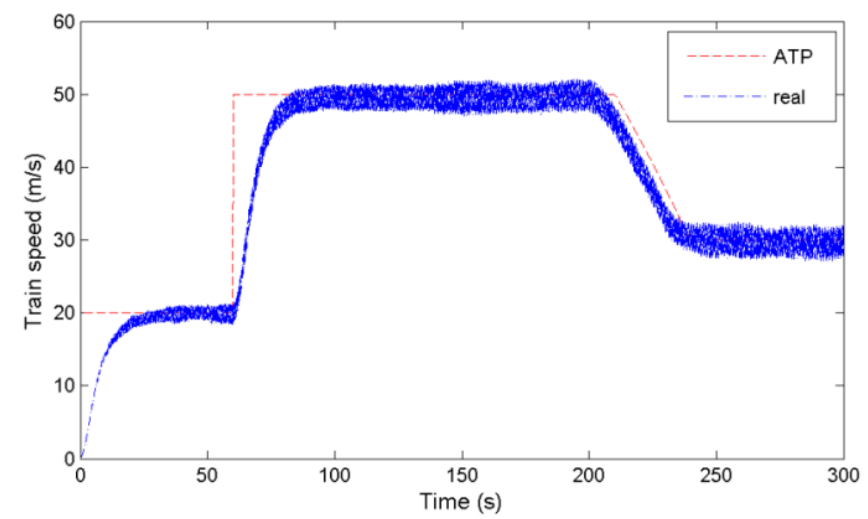

Figure 3. Train Speed Trajectory under Case B without Constraints Tightening

Force in Figure 5 will change a little in order to compensate the robust performance. Therefore, the train can run normally under the train protection system using the Algorithm 2 despite of the bounded disturbances. In the meantime, note that the in-train force (Table 3) is much bigger than in case A. Fortunately, they are all in the constraint of maximum in-train force.

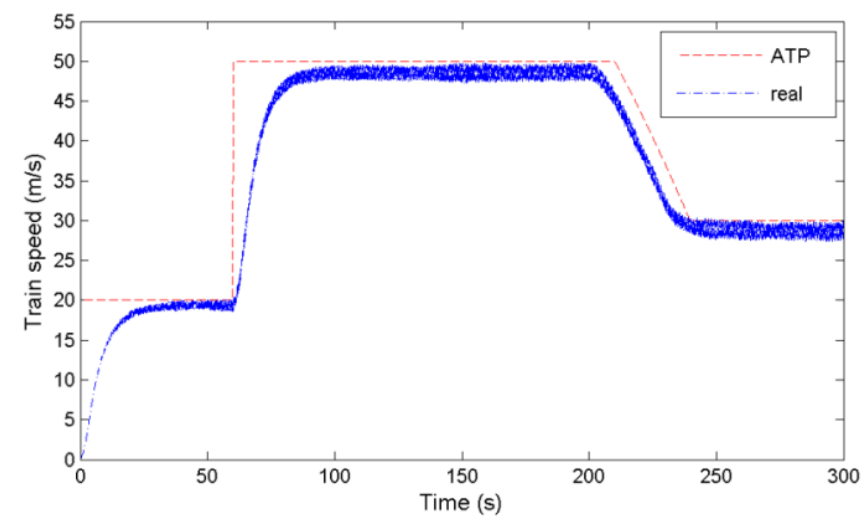

Figure 4. Train Speed Trajectory under Case B with Constraints Tightening 

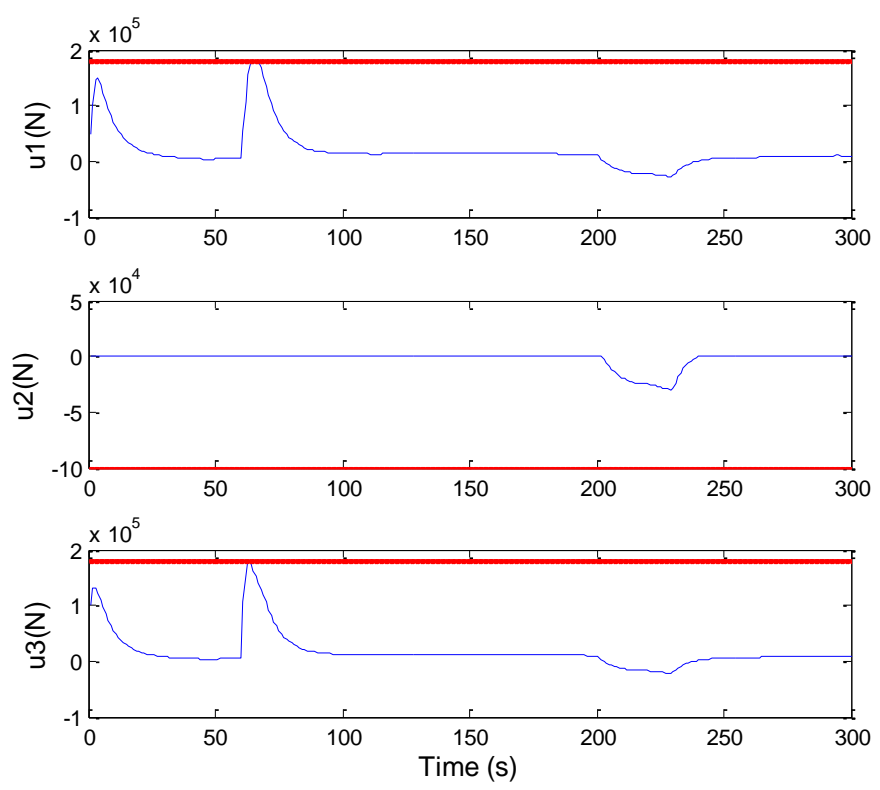

Figure 5. Traction/Braking Force under Case B

Table 3. In-Train Force under Case B

\begin{tabular}{c|c|c|c}
\hline \hline & Mean & Max & Min \\
\hline$f_{\text {in }_{1}}[k N]$ & 4.8 & 940 & -880 \\
\hline$f_{\text {in. }_{2}}[k N]$ & -8.5 & 860 & -810 \\
\hline \hline
\end{tabular}

\section{Conclusion}

This work introduces an algorithm of automatic train operation system which combines the high-level (optimal level) with the low-level (tracking level) together in the presence of model disturbance and hard constraints. Compared with the traditional approach which calculates the trajectory first by optimization then tracks it using a closed-loop controller, the proposed algorithm calculates an artificial reference for tracking and uses constraints tightening for robustness, which not only guarantees the robustness but also simplifies the complexity of the computation. Simulation results verify that the train can run in a speed closed to the ATP curve without exceeding despite of the external disturbance.

\section{Appendix.}

\section{Proof of Theorem 1}

In order to prove the stability of the proposed controller under Algorithm 2, the feasibility must to be guaranteed firstly. Then the robust convergence of Algorithm 2 that combines the piecewise constant reference tracking and constraint tightening is proved.

\section{Feasibility:}

Assume that at some time step $k$, the optimal solution of problem $P_{N}\left(x_{k}, \hat{\mathbf{x}}_{s}, \mathcal{Y}, \mathcal{W}\right)$ is $\left(\mathbf{u}^{0}(k), \theta^{0}(k)\right)$ and correspondingly the optimal state and constraint output are $\mathbf{x}^{0}(k)$ and $\mathbf{y}^{0}(k)$ respectively, where 


$$
\begin{aligned}
& \mathbf{u}^{0}(k):=\left\{\mathbf{u}^{0}(0 ; k), \mathbf{u}^{0}(1 ; k), \cdots, \mathbf{u}^{0}(N-1 ; k)\right\} \\
& \mathbf{x}^{0}(k):=\left\{\mathbf{x}^{0}(0 ; k), \mathbf{x}^{0}(1 ; k), \cdots, \mathbf{x}^{0}(N-1 ; k)\right\} \\
& \mathbf{y}^{0}(k):=\left\{\mathbf{y}^{0}(0 ; k), \mathbf{y}^{0}(1 ; k), \cdots, \mathbf{y}^{0}(N-1 ; k)\right\}
\end{aligned}
$$

Then consider $\theta(k+1)=\theta^{0}(k)$ and construct a candidate solution $(\hat{\mathbf{u}}(k+1), \theta(k+1))$ at time step $k+1$, which is expressed as:

$$
\hat{\mathbf{u}}(k+1)=\{\hat{\mathbf{u}}(0 ; k+1), \hat{\mathbf{u}}(1 ; k+1), \cdots, \hat{\mathbf{u}}(N-1 ; k+1)\}
$$

where

$$
\begin{gathered}
\hat{\mathbf{u}}(j ; k+1)=\mathbf{u}^{0}(j+1 ; k)+K(j) L(j) w(k), \quad(\forall j=0,1, \cdots, N-2) \\
\hat{\mathbf{u}}(N-1 ; k+1)=\kappa\left(x^{0}(N ; k), \theta^{0}(k)\right), \\
\hat{\mathbf{x}}(j ; k+1)=\mathbf{x}^{0}(j+1 ; k)+L(j) w(k), \quad(\forall j=0,1, \cdots, N-1) \\
\hat{\mathbf{x}}(N ; k+1)=A \mathbf{x}^{0}(N ; k)+B \kappa\left(x^{0}(N ; k), \theta^{0}(k)\right) \\
\hat{\mathbf{y}}(j ; k+1)=C \hat{\mathbf{x}}(j ; k+1)+D \hat{\mathbf{u}}(j ; k+1), \quad(\forall j=0,1, \cdots, N-1)
\end{gathered}
$$

In order to prove the feasibility of candidate solution, it is necessary to confirm that the candidate input, state and constraint output at time step $k+1$ meets all the constraint requests (22) despite of the disturbance $\mathbf{w}(k) \in \mathcal{W}$.

\section{- Dynamics constraints:}

Due to the optimal state and input satisfy the dynamics constraints in (22.a) at time $k$, the equation

$$
\mathbf{x}^{0}(j+2 ; k)=A \mathbf{x}^{0}(j+1 ; k)+B \mathbf{u}^{0}(j+1 ; k), \quad(\forall j=0,1, \cdots, N-2)
$$

is true. Then substitute (31.a) and (31.c) into it, we could find that:

$$
\begin{aligned}
\hat{\mathbf{x}}(j+1 ; k+1)-L(j+1) w(k)= & A(\hat{\mathbf{x}}(j ; k+1)-L(j) w(k)) \\
& +B(\hat{u}(j ; k+1)-K(j) L(j) w(k)) \\
= & A(\hat{\mathbf{x}}(j ; k+1)+B(\hat{u}(j ; k+1) \\
& -(A+B K(j)) L(j) w(k)
\end{aligned}
$$

In the meantime, we know the definition of $L(k)$, that is $L(j+1)=(A+B K(j)) L(j)$.

Hence, $\quad \hat{\mathbf{x}}(j+1 ; k+1)=A \hat{\mathbf{x}}(j ; k+1)+B \hat{u}(j ; k+1), \quad(\forall j=0,1, \cdots, N-2)$. On the other hand, it can also be proven when $j=N-1$ due to the property of constructed candidate solution (31.b) and (31.d). Therefore, the candidate solution satisfies the dynamics constraints (22.a) at time $k+1$ for all $j=0,1, \cdots, N-1$.

\section{- Initial constraints:}

It is obvious that, at time $k+1$, the real state $\mathbf{x}(k+1)$ is:

$$
\mathbf{x}(k+1)=\mathbf{x}^{0}(1 ; k)+w(k)
$$

As well, substitute $L(0)=\mathbf{I}$ into the equation (31.c), get:

$$
\hat{\mathbf{x}}(0 ; k+1)=\mathbf{x}^{0}(1 ; k)+w(k)
$$

Therefore, $\hat{\mathbf{x}}(0 ; k+1)=\mathbf{x}(k+1)$, which satisfies the initial constraint (22.c) at time $k+1$. 


\section{- Terminal constraints:}

When $j=N-1$, the equation (31.c) is expressed as:

$$
\hat{\mathbf{x}}(N-1 ; k+1)=\mathbf{x}^{0}(N ; k)+L(N-1) w(k)
$$

As the optimal state satisfies the terminal constraint (22.d) at time $k$, $\mathbf{x}^{0}(N ; k) \in \mathcal{X}_{F}$ and the property of Pontryagin difference and the definition of constraint tightening terminal set (26),

$$
\hat{\mathbf{x}}(N-1 ; k+1) \in \mathcal{R}
$$

And use equation (27) and (31.b), we have

$$
A \hat{\mathbf{x}}(N ; k)+B \kappa\left(\hat{\mathbf{x}}(N ; k), \theta^{0}(k)\right)+L(N-1) w(k) \in \mathcal{R}, \quad \forall w \in \mathcal{W}
$$

After that, substitute (31.d) into the equation above,

$$
\hat{\mathbf{x}}(N ; k+1)+L(N-1) w(k) \in \mathcal{R}, \quad \forall w \in \mathcal{W}
$$

equivalently, $\quad \hat{\mathbf{x}}(N ; k+1) \in \mathcal{R} \sim L(N) \mathcal{W}(k), \quad \forall w \in \mathcal{W}$. After using the definition of robust invariant set, we have

$$
\hat{\mathbf{x}}(N ; k+1) \in \mathcal{X}_{F}
$$

Therefore, combining the assumption $\theta(k+1)=\theta^{0}(k)$, the terminal state $(\hat{\mathbf{x}}(N ; k+1), \theta(k+1)) \in \mathcal{X}_{F}^{z}$, which satisfies the terminal constraint (22.d).

- Output constraints:

Based on the equation (31.a), (31.c) and (31.e), it is easy to find that

$$
\hat{\mathbf{y}}(j ; k+1)=\mathbf{y}^{0}(j+1 ; k)+(C+D K(j)) L(j) w(k), \quad(\forall j=0,1, \cdots, N-2)
$$

Moreover because of the feasibility at time $k$

$$
\mathbf{y}^{0}(j+1 ; k) \in \mathcal{Y}(j+1), \quad(\forall j=0,1, \cdots, N-2),
$$

the definition of the output constraints sets (23) and the property of the Pontryagin difference, we have

$$
\mathbf{y}^{0}(j+1 ; k)+(C+D K(j)) L(j) w(k) \in \mathcal{Y}(j),(\forall w \in \mathcal{W}, j=0,1, \cdots, N-2)
$$

Therefore,

$$
\hat{\mathbf{y}}(j ; k+1) \in \mathcal{Y}(j), \quad(\forall j=0,1, \cdots, N-2)
$$

And it is easy to confirm that the final constraint output also satisfies (22.b).

Therefore, the candidate solution meet the output constraint at time $k+1$.

In summary, based on the proof above we know if the optimal problem is feasible at time $k$, there exist a candidate solution is also feasible at time $k+1$. Hence if the algorithm is feasible at initial time, it is feasible for all the future times by recursion.

\section{Robust Convergence:}

For simplicity, use these notations in the proof

$$
\begin{aligned}
V_{N}^{0}(k) & =V_{N}^{0}\left(x(k), \hat{\mathbf{x}}_{s}\right) \\
V_{N}^{0}(k+1) & =V_{N}^{0}\left(x(k+1), \hat{\mathbf{x}}_{s}\right) \\
\hat{V}_{N}(k+1) & =\hat{V}_{N}\left(x(k+1), \hat{\mathbf{x}}_{s}, \hat{\mathbf{u}}, \theta(k+1)\right)
\end{aligned}
$$

Based on the definition of cost function (20), the optimal cost at time $k$ is written as: 


$$
V_{N}^{0}(k)=\sum_{j=0}^{N-1}\left\{\left\|\mathbf{u}^{0}(j ; k)\right\|_{R}+\left\|\mathbf{x}^{0}(j ; k)\right\|_{Q}\right\}+\left\|x(N ; k)-x_{s}(k)\right\|_{P}^{2}+\left\|x_{s}(k)-\hat{x}_{s}\right\|_{T}^{2}
$$

So the cost function for the candidate solution at time $k+1$ is:

$$
\begin{aligned}
\hat{V}_{N}(k+1)= & \sum_{j=0}^{N-1}\left\{\|\hat{\mathbf{u}}(j ; k+1)\|_{R}+\|\hat{\mathbf{x}}(j ; k+1)\|_{Q}\right\}+\left\|x(N ; k+1)-x_{s}(k+1)\right\|_{P}^{2} \\
& +\left\|x_{s}(k+1)-\hat{x}_{s}\right\|_{T}^{2} \\
= & \sum_{j=0}^{N-2}\left\{\left\|\mathbf{u}^{0}(j+1 ; k)+K(j) L(j) w(k)\right\|_{R}+\left\|\mathbf{x}^{0}(j+1 ; k)+L(j) w(k)\right\|_{Q}\right\} \\
+ & \left\|\mathbf{x}^{0}(N ; k)\right\|_{Q}+\left\|\kappa\left(\mathbf{x}^{0}(N ; k), \theta^{0}(k)\right)\right\|_{R}+\left\|x(N ; k+1)-x_{s}(k+1)\right\|_{P}^{2} \\
& +\left\|x_{s}(k+1)-\hat{x}_{s}\right\|_{T}^{2}
\end{aligned}
$$

then use the triangle inequality,

$$
\begin{aligned}
\hat{V}_{N}(k+1) \leq & \sum_{j=0}^{N-2}\left\{\left\|\mathbf{u}^{0}(j+1 ; k)\right\|+\|K(j) L(j) w(k)\|_{R}+\left\|\mathbf{x}^{0}(j+1 ; k)\right\|+\|L(j) w(k)\|_{Q}\right\} \\
& +\left\|\mathbf{x}^{0}(N ; k)\right\|_{Q}+\left\|k\left(\mathbf{x}^{0}(N ; k), \theta^{0}(k)\right)\right\|_{R}+\left\|x(N ; k+1)-x_{s}(k+1)\right\|_{P}^{2} \\
& +\left\|x_{s}(k+1)-\hat{x}_{s}\right\|_{T}^{2}
\end{aligned}
$$

After that use the standard method for the stability proofs of MPC [10], we have $\hat{V}_{N}(k+1) \leq V^{0}(\mathbf{x}(k))-\left\|x(k)-x_{s}^{0}(k)\right\|_{Q}+\sum_{j=0}^{N-1}\left\{\|K(j) L(j) w(k)\|_{R}+\|L(j) w(k)\|_{Q}\right\}$ As the definition of $\alpha$ and the property of $V^{0}(k+1)$ and $\hat{V}(k+1)$,

$$
\begin{aligned}
V^{0}(k+1) & \leq \hat{V}(k+1) \\
& \leq V^{0}(k)-\left\|x(k)-x_{s}^{0}(k)\right\|_{Q}+\alpha
\end{aligned}
$$

Finally use Lemma 3 of the paper [15], we have

$$
V^{0}(k+1) \leq V^{0}(k)-\left\|x(k)-\hat{x}_{s}\right\|_{T}+\alpha
$$

Therefore, if the state is outside the convergence set $\mathcal{X}_{c}$, that is $-\left\|x(k)-\hat{x}_{s}\right\|_{T}+\alpha \leq 0$ which implies that the optimal cost function is strictly decreasing. However, in practice there exit a minimum value of the optimal cost function. Hence the state will not always be out the set $\mathcal{X}_{c}$, which means it will enter the set $\mathcal{X}_{c}$ at some time.

In conclusion, the feasibility and robust convergence are both be proven, therefore the stability of Algorithm 2 is guaranteed.

\section{Acknowledgements}

This work was supported by the Fundamental Research Funds for the Central Universities (2014JBM019).

\section{References:}

[1] P.G. Howlett, I.P. Milroy and P.J. Pudney, "Energy-efficient train control. Control Engineering Practice", 2(2): p. 193-200 (1994).

[2] R. Liu and I.M. Golovitcher, "Energy-efficient operation of rail vehicles", Transportation Research Part A: Policy and Practice, 37(10): p. 917-932 (2003).

[3] A.R. Albrecht, P.G. Howlett, P.J. Pudney and X. Vu, "Energy-efficient train control: From local convexity to global optimization and uniqueness", Automatica, 49(10): p. 3072-3078 (2013). 
[4] S. Shuai, T. Tao, L. Xiang and G. Ziyou, "Optimization of Multitrain Operations in a Subway System", Intelligent Transportation Systems, IEEE Transactions on. 15(2): p. 673-684 (2014).

[5] Chou, M. and X. Xia, Optimal cruise control of heavy-haul trains equipped with electronically controlled pneumatic brake systems. Control Engineering Practice. 15(5): p. 511-519 (2007).

[6] C.D. Yang and Y.P. Sun, "Mixed H2/H cruise controller design for high speed train", International Journal of control, 74(9): p. 905-920\%@0020-7179 (2001).

[7] Q. Song, Y.D. Song, T. Tang and D. Ning, "Computationally Inexpensive Tracking Control of High-Speed Trains With Traction/Braking Saturation", Ieee Transactions on Intelligent Transportation Systems, 12(4): p. 1116-1125 (2011).

[8] S.H. Heqing, H. Zhongsheng and L. Dayou, "Coordinated Iterative Learning Control Schemes for Train Trajectory Tracking With Overspeed Protection", Automation Science and Engineering, IEEE Transactions on 10(2): p. 323-333 (2013).

[9] G. Shigen, D. Hairong, C. Yao, N. Bin, C. Guanrong and Y. Xiaoxia, "Approximation-Based Robust Adaptive Automatic Train Control: An Approach for Actuator Saturation”, Intelligent Transportation Systems, IEEE Transactions on. 14(4): p. 1733-1742 (2013).

[10] J.M. Maciejowski, "Predictive control: with constraints", Pearson education (2002).

[11] A. Richards and J. How, "Robust stable model predictive control with constraint tightening", in American Control Conference, 2006.

[12] I. Kolmanovsky and E.G. Gilbert, "Theory and computation of disturbance invariant sets for discrete-time linear systems", Mathematical Problems in Engineering, 4(4): p. 317-367 (1998).

[13] M. Chou, X. Xia and C. Kayser, "Modelling and model validation of heavy-haul trains equipped with electronically controlled pneumatic brake systems", Control Engineering Practice, 15(4): p. 501-509 (2007).

[14] M. Bahadorian, B. Savkovic, R. Eaton and T. Hesketh, "Robust Model Predictive Control for automated trajectory tracking of an Unmanned Ground Vehicle", in American Control Conference (ACC), 2012.

[15] D. Limon, I. Alvarado, T. Alamo and E.F. Camacho, "MPC for tracking piecewise constant references for constrained linear systems", Automatica, 44(9): p. 2382-2387 (2008).

[16] Gossner, J.R., B. Kouvaritakis, and J.A. Rossiter, "Stable generalized predictive control with constraints and bounded disturbances", Automatica, 33(4): p. 551-568 (1997).

[17] R. Zhong, "Train traction calculation", 3th edition: China Railway Publishing House (2012).

[18] M. Herceg, M. Kvasnica, C.N. Jones and M. Morari, "Multi-Parametric Toolbox 3.0. in Control Conference (ECC)", 2013 European.

[19] E.C. Kerrigan, "Robust constraint satisfaction: Invariant sets and predictive control", (2000), PhD thesis, Cambridge.

\section{Author}

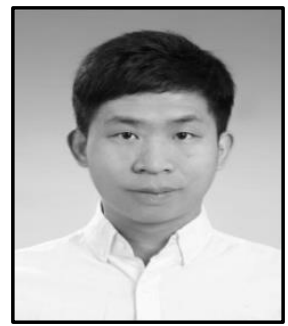

Longsheng Wang received the B.S. degree from Beijing Jiaotong University, Beijing, China, in 2010. He is currently working toward the Ph.D. degree with the School of Electronics and Information Engineering, Beijing Jiaotong University. His current research interests include automatic train operation, model predictive control and adaptive control. 
International Journal of Control and Automation Vol.8, No.11 (2015) 